\title{
Hippocampal subfield volumes: age, vascular risk, and correlation with associative memory
}

\section{Yee Lee Shing ${ }^{1,2 *}$, Karen M. Rodrigue ${ }^{3}$, Kristen M. Kennedy ${ }^{3}$, Yana Fandakova' , Nils Bodammer', Markus Werkle-Bergner ${ }^{1}$, Ulman Lindenberger ${ }^{1}$ and Naftali Raz ${ }^{4}$}

\author{
' Center for Lifespan Psychology, Max Planck Institute for Human Development, Berlin, Germany \\ 2 Department of Psychology, Humboldt University, Berlin, Germany \\ ${ }^{3}$ Center for Vital Longevity, School of Behavioral and Brain Sciences, University of Texas at Dallas, Dallas, TX, USA \\ ${ }_{4}^{4}$ Institute of Gerontology, Wayne State University, Detroit, MI, USA
}

\section{Edited by:}

P. Hemachandra Reddy, Oregon Health and Science University, USA

\section{Reviewed by:}

D. Samba Reddy, Texas A\&M Health

Science Center, USA

Asgar Zaheer, University of lowa

Hospitals and Clinics, USA

*Correspondence:

Yee Lee Shing, Center for Lifespan Psychology, Max Planck Institute for

Human Development, Lentzeallee 94, 14195 Berlin, Germany.

e-mail:yshing@mpib-berlin.mpg.de
Aging and age-related diseases have negative impact on the hippocampus $(\mathrm{HC})$, which is crucial for such age-sensitive functions as memory formation, maintenance, and retrieval. We examined age differences in hippocampal subfield volumes in 10 younger and 19 older adults, and association of those volumes with memory performance in the older participants. We manually measured volumes of $\mathrm{HC}$ regions $\mathrm{CA} 1$ and $\mathrm{CA} 2$ (CA1-2), sectors CA3 and CA4 plus dentate gyrus (CA3-4/DG), subiculum, and the entorhinal cortex using a contrast-optimized high-resolution PD-weighted MRI sequence. Although, as in previous reports, the volume of one region (CA1-2) was larger in the young, the difference was due to the presence of hypertensive subjects among the older adults. Among older participants, increased false alarm rate in an associative recognition memory task was linked to reduced CA3-4/DG volume. We discuss the role of the $D G$ in pattern separation and the formation of discrete memory representations.

Keywords: aging, hippocampus, MRI, dentate gyrus, memory, pattern separation

\section{INTRODUCTION}

Episodic memory declines with age (Verhaeghen et al., 1993), and one of its important substrates, the hippocampal formation (Squire, 1987) is sensitive to aging and age-related pathology (Jack et al., 2000; Raz et al., 2005). However, the link between age-related memory deficits and changes in the hippocampal stucture is unclear (Van Petten, 2004; Raz and Rodrigue, 2006). A meta-analysis of the extant literature demonstrates that such connection probably does not exist among the young adults but may emerge in samples with higher proportion of older participants (Van Petten, 2004). One of the major problems and probable sources of inconsistency among the studies may be insufficient spatial resolution of the measurements. The hippocampus (HC) is a heterogeneous structure comprised of cytoarchitectonically distinct regions: the subiculum, the four cornu ammonis sectors (CA1-4), and the dentate gyrus (DG; Duvernoy, 2005). The adjacent part of the hippocampal formation, entorhinal cortex (ERC) connects to the HC proper via the perforant path (PP). Whereas regional $\mathrm{HC}$ anatomy has been well researched in animals (e.g., Lister and Barnes, 2009), until recently, its cytoarchitectonic heterogeneity presented a challenge for in vivo measurement in humans.

Although age- and pathology-related structural differences in $\mathrm{HC}$ subfields were examined by semi-automated procedures (Burggren et al., 2008; Chételat et al., 2008; Apostolova et al., 2010; Yassa et al., 2010b), only one research group reported manual measures (Mueller et al., 2007). Manual regional morphometry of HC revealed a significant reduction of CA1 volume in healthy older adults (Mueller et al., 2007). This association was replicated in two subsequent studies with additional reliable negative effects of age on the region defined as CA3/4\&DG (Mueller et al., 2008; Mueller and Weiner, 2009). However, there are no reports of replication by other research groups. Moreover, it is unclear whether age-related factors such as vascular risk (Franklin et al., 1997) contribute to the age differences, as its effects on $\mathrm{HC}$ sub-structures have not been taken into account in previous studies. It is plausible, for example, that hypertension, which accelerates hippocampal shrinkage (Raz et al., 2005) and impairs cerebral perfusion (Levy et al., 2008) may differentially affect aging of $\mathrm{HC}$ regions (e.g., $\mathrm{CA1}$ ) known for their heightened vulnerability to ischemia (Zola-Morgan et al., 1986).

Even when regional age differences in HC structure are observed in healthy adults functional implications of such findings are unclear, as there are almost no studies correlating such variations with memory performance. Persons with documented memory impairment have smaller CA1 and DG/ CA3 regions than age-matched controls (Yassa et al., 2010b), and focal ischemic lesions in CAl sector of HC are linked to impaired acquisition of a spatial navigation skill and place memory (Bartsch et al., 2010). However, the relative contribution of volume differences in the HC subfields to memory of healthy adults remains unknown.

The goals of the current study were to examine age-related regional volume differences in the HC subfields, to gauge the contribution of vascular risk to those differences, and to evaluate strength of associations between the volume of HC subfields and associative episodic memory in healthy older adults. We focused on association memory because memory deficits in the target population involve failures of binding multiple pieces of information with their contexts (Spencer and Raz, 1995; Old and Naveh-Benjamin, 2008; Shing et al., 2009) and excess of false recollection (Jacoby and Rhodes, 2006; Shing et al., 2008). 


\section{MATERIALS AND METHODS PARTICIPANTS}

Participants included 19 older (ages 70-78 years, $M=75.4, \mathrm{SD}=2.9$, eight women) and 10 younger adults (ages 20-25 years, $M=23$, $\mathrm{SD}=1.7$, five women). The participants were screened for history of major neurological or psychiatric disorders, head trauma, and present contraindications to an MRI examination. Ten of the older adults reported having hypertension controlled by antihypertensive medication, and one of them also reported having diabetes. All participants except one were right handed, and had normal or corrected-to-normal visual and auditory acuity. As expected, younger adults performed significantly faster than older participants on a measure of perceptual speed (Digit Symbol; cf. Wechsler, 1955): $t(25)=7.13, p<0.01$. Older adults were also screened for cognitive impairment with the Mini Mental State Examination (MMSE, $M=28.8, \mathrm{SD}=1.4$, Range $=26-30$ ).

\section{MRI PROCEDURE}

The images were acquired on a Siemens 3 Tesla TIM Trio scanner (Siemens Medical AG, Erlangen, Germany). A high-resolution MP-RAGE sequence $(\mathrm{TR}=2500 \mathrm{~ms} ; \mathrm{TE}=4.76 \mathrm{~ms} ; \mathrm{TI}=1100 \mathrm{~ms}$; flip angle $=7^{\circ}$; one average; matrix $176 \times 256 \times 256$; voxel size $1 \mathrm{~mm} \times 1 \mathrm{~mm} \times 1 \mathrm{~mm}$ ) was acquired in the beginning of the session for determining scanning orientation and measuring intracranial volume (ICV). After that, we acquired a high-resolution PD-weighted turbo spin echo (TSE) sequence $(\mathrm{TE}=16 \mathrm{~ms}$, $\mathrm{TR}=7000 \mathrm{~ms}$; in-plane resolution $=0.4 \mathrm{~mm} \times 0.4 \mathrm{~mm}$; slice thickness $=2 \mathrm{~mm}$; turbo factor 11 , two averages, 30 interleaved slices perpendicular to the principal axis of the right $\mathrm{HC})$. We acquired two TSE sequences, which enabled rough motion correction by co-registering these series as the first processing step.

\section{BEHAVIORAL PROCEDURE}

Only the 19 older adults underwent behavioral testing; 11 of them had participated in an earlier behavioral study (Shing et al., 2008, 2009), and additional eight were later recruited specifically for this study. The delay between behavioral testing and scanning was 2 years for the 11 participants, and 2 weeks for the eight who were recruited later. Behavioral testing procedure, an associative recognition memory task (Shing et al., 2008), was identical for all participants. During the encoding phase of the task, 45 German word pairs were presented sequentially, for $6 \mathrm{~s}$ each. Participants were instructed to study the word pairs for an impending recognition test. After the encoding phase, participants counted backward by threes for $90 \mathrm{~s}$ as an interpolated activity aimed to prevent rehearsal.

At retrieval, 60 memory probes were presented sequentially and participants decided whether they had seen each probe at the encoding phase (old-new judgment). Thirty of the probes consisted of intact pairs from the encoding phase. Fifteen probes were rearranged lure pairs of recombined words from different study pairs at encoding; 15 probes were new-new lure pairs of words that never appeared before. Upon recognition of a pair from the encoding set, the participants were instructed to press the button labeled "old." Participants were informed about the two types of lures and were instructed to press the button labeled "new" upon recognizing those. To assess the accuracy of recognition, we used the difference between proportion of correctly recognized old pairs (hit rate, HR) and proportion of incorrectly recognized rearranged pairs [false alarm (FA) rate; Snodgrass and Corwin, 1988]. Note that rejecting the rearranged pairs that contained familiar words was more difficult than rejecting the new-new pairs.

\section{POST-PROCESSING AND MEASUREMENT OF THE HIPPOCAMPAL SUBFIELDS}

In demarcation of HC regions, we followed Mueller et al. (2007, 2008 ) with slight modifications. Two independent raters (Kristen M. Kennedy, Karen M. Rodrigue), who were blind to the participants' sex and age, used the Analyze software (Mayo Clinic, Rochester, MN, USA) and a 21 -in digital tablet with a stylus (Wacom Cintiq) to trace all images. The traced regions included ERC, subiculum, CA1-2, and CA3-4\&DG. All subfields were traced bilaterally, and the ranges were allowed to differ by starting/ending slice based on each hemisphere's anatomy. The tracing (see an example in Figure 1) started on the first slice on which the head of the HC was no longer visible.

\section{Entorhinal cortex}

The ERC was traced on six coronal slices from the end of the subiculum to the opening of the collateral sulcus. Tracing of the ERC began five slices anterior to the starting slice; the range was increased compared to Mueller et al's (2007) method, as the region was identifiable on six slices.

\section{CA3-4\&dentate gyrus (DG)}

The CA3 and CA4 subfields were combined to increase reliability. The DG was traced with a line drawn just inside the high-contrast dark circular boundary of the dentate. This region was traced on the starting slice and the two slices posterior to it.

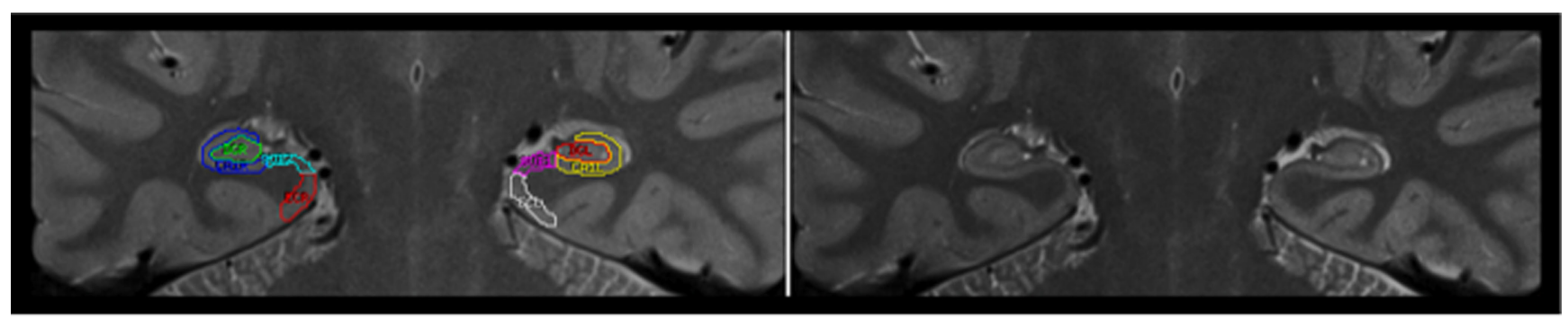

FIGURE 1 | Example of hippocampal subfields tracing. A starting slice with (left side) and without (right side) tracing is shown. 


\section{CA1-2 (CA1)}

This region was traced on the same three coronal slices as the DG. Unlike Mueller et al. (2007), we included the CA2 with the CA1 ROI due to poor anatomical boundaries for defining the CA2 subfield. CA1 was traced from the dorsal border around the DG to where it meets the subiculum medially.

\section{Subiculum (SUB)}

The left and right SUB were traced on three coronal slices beginning on the same starting slice as CA1 and DG. Tracing began at the end of CAl and proceeded to the dorsal entorhinal boundary determined by a diagonal line drawn as an extension of the medial DG border.

To account for differences in head size, ICV were measured from T1-weighted images and ICV was used to adjust subfield volumes via ANCOVA (as described in Raz et al., 2010). Volumes were computed by multiplying the sum of slices area by slice thickness $(2 \mathrm{~mm})$. Inter-rater reliability (intra-class correlation for random raters, ICC formula 2; Shrout and Fleiss, 1979) was very good: ERC ICC $=0.94 ;$ CA1 -2 ICC $=0.99 ;$ CA3-\&DG ICC $=0.89$, and subiculum ICC $=0.89$.

\section{RESULTS}

\section{AGE DIFFERENCES IN HC SUBFIELD VOLUME}

Data for each group were checked for outliers and violations of normality. Only one observation from the older group (CA1-2) was an outlier (volume $=499.72 \mathrm{~mm}^{3}$, two standard deviations above the group mean, $M_{\text {older adults }}=356.63 \mathrm{~mm}^{3}$ ). After excluding this observation, neither deviations from normality nor significant outliers remained. No laterality difference was found for the subfield volumes, except for CA3-4\&DG. Right CA3-4\&DG was larger than their left counterparts, but there was no significant age $\times$ hemisphere interaction $(F<1)$. Therefore, sums of left and right volumes in each subfield were used in all further analyses.

To examine excessive leverage of the outlier, we used bootstrapping to evaluate age-group differences in subfield volumes and correlations between memory performance and volumetric measures. For each bootstrap analysis, we drew 5000 random samples with replacement, the same size as the original sample, and computed the statistic of interest for each. The resulting values were sorted in ascending order. To construct $95 \%$ confidence intervals (95\% $\mathrm{CI}$ ), we used the 2.5 and 97.5 percentiles from the bootstrap distribution. To examine the influence of the observation identified as potentially outlying, the bootstrapping was conducted with and without that participant's data. Age differences in volumes were deemed reliable if the corresponding $t$-value for the original sample fell outside the bootstrapped 95\% CI. Associations between subfield volumes and memory performance were regarded as statistically reliable if the bootstrapped $95 \%$ CI for the correlation coefficients did not include zero.

In one-way ANOVAs for each subfield volume, significant age differences were found only in CA1-2: $t(26)=2.14, p=0.04$, see Figure 2. Bootstrap analyses confirmed that CA1-2 was the only region showing a reliable age difference. The bootstrap analysis without the outlier resulted in a reliable difference between the age groups, $p=0.02$, whereas its inclusion resulted in a marginally reliable effect, $p=0.06$.

\section{HYPERTENSION EFFECTS ON HC SUBFIELD VOLUME}

To examine the contribution of vascular risk (hypertension) to the observed age differences, we compared regional $\mathrm{HC}$ volumes in hypertensive and normotensive older adults. Although all four examined regions showed reduced volume in the hypertensive group, it was statistically significant only in CA1-2: $\mathrm{F}(1,16)=5.39$, $p=0.034$. The results of this analysis are illustrated in Figure 3 . Bootstrap follow-up analysis confirmed that CA1-2 was the only region showing a reliable difference attributable to hypertension, $p=0.05$. Furthermore, bootstrapping showed that normotensive older adults did not differ from younger adults in their CA1-2 volume, $p=0.60$.

\section{HC SUBFIELD VOLUME ASSOCIATIONS WITH MEMORY}

Associations of hippocampal subfield volumes and associative memory performance were examined in older participants only, as behavioral data for younger adults were unavailable. We observed a positive correlation between CA3-4\&DG and memory accuracy $(r=0.53, p=0.02)$, indicating that better associative recognition memory was linked to larger volume of that region (see Figure 4A). No other correlation was significant (see Table 1), suggesting that the observed association was selective to this region. Analyzing HR and FA separately, we found that the correlation between CA3$4 \& D G$ and memory accuracy was mainly driven by FA (Figure 4B). In other words, the larger the volume on CA3-4\&DG, the lesser the likelihood of committing a FA $(r=-0.56, p=0.02)$. Bootstrapping confirmed these conclusions as none of the $95 \% \mathrm{CI}$ for the correlations of interest included zero. The results remained unaffected by inclusion or exclusion of the CA1-2 outlier.

We tested whether there was an effect of difference in delay between cognitive assessment and scanning ( 2 weeks vs. 2 years). The magnitude of the correlations remained similar within each group, but became non-significant due to small sample size.

To examine the specificity of the association between regional volume and memory, we examined the correlations of the subfield volumes with other cognitive variables, including perceptual speed and MMSE (see Table 1). Only one correlation, between CA1-2 volume and MMSE, was significant: $r=0.48, p<0.05$. Bootstrapping showed that this correlation was robust as its $95 \%$ CI did not included zero. Partialing out age did not result in a substantial change of the correlation: $r=0.46, p=0.06$. Adopting more stringent MMSE cut-offs of 27 and 28 does not affect the results: $r=0.49, p=0.047$ and $r=0.46, p=0.073$.

\section{DISCUSSION}

The main finding of this study is the association between smaller volumes of the CA3-4\&DG region of the HC and increased false recognition memory in older adults. To the best of our knowledge, this is the first report of an association between regional $\mathrm{HC}$ volume and episodic memory in a healthy population. These results concur with the report of memory deficits in primates with reduced DG cerebral blood flow (Small et al., 2004) and the findings of smaller CA3/dentate volumes of persons with memory problems (Yassa et al., 2010b). Our observations are also in accord with the link between reduced integrity of the major input pathway from the ERC to the hippocampal formation, the PP, and memory in older adults (Yassa et al., 2010a). 

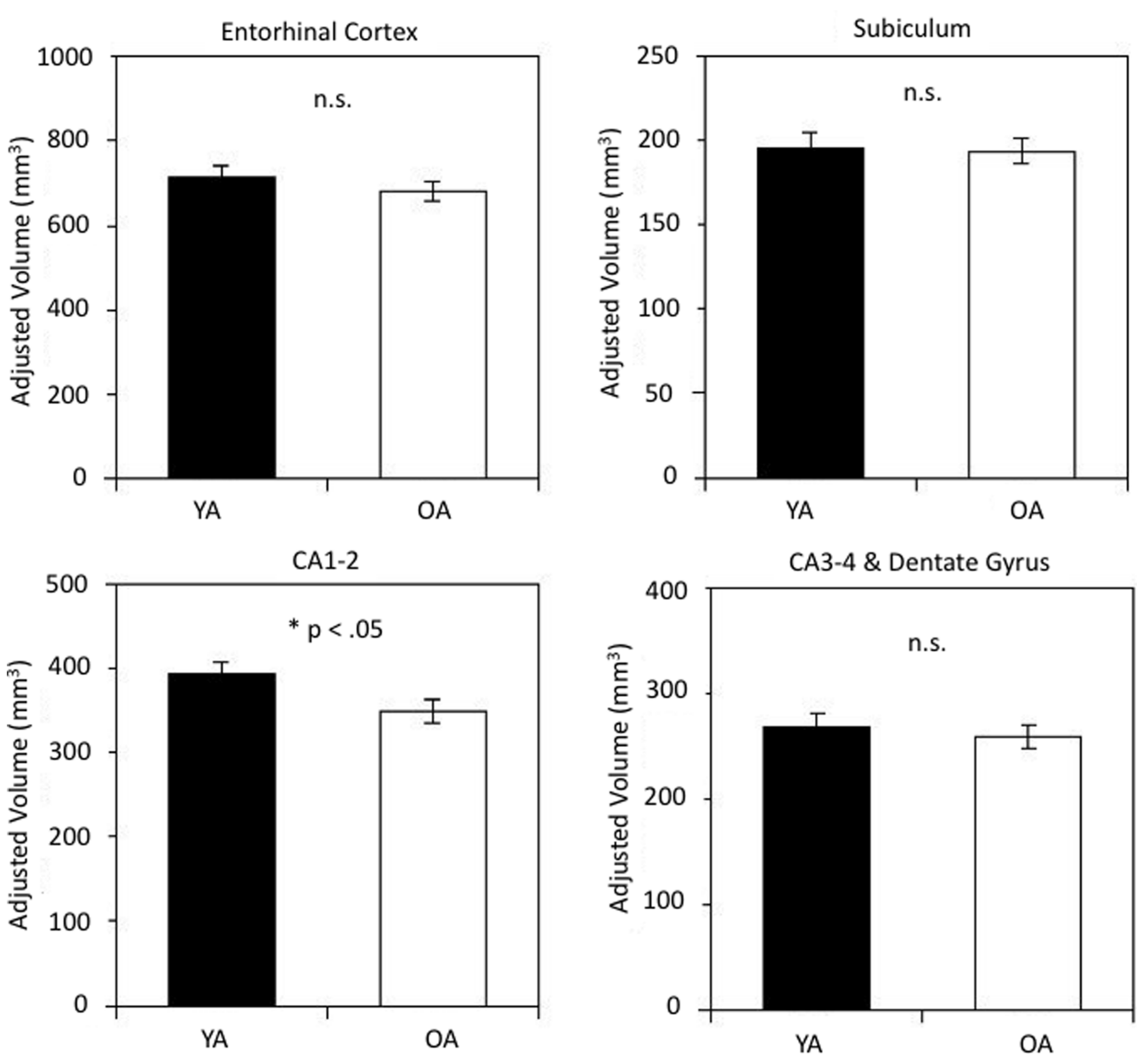

FIGURE 2 | Hippocampal subfield volumes in younger and older adults. Age-group differences in volume were reliable for subfield CA1-2. Volumes are adjusted for intracranial volume (ICV) as detailed in the text. The bars represent SE of the mean.

Thus, we highlight the importance of structural integrity of the CA3-4\&DG region in the context of recognizing changes (rearranged lures) in familiar stimuli.

In addition, we observed smaller volumes of CA1-2 in older adults, replicating the reported pattern of regional volume differences in HC (Mueller et al., 2007, 2010). However, by taking into account the hypertension diagnosis of some of the participants, we show that age differences in CA1 may reflect vulnerability to vascular risk rather than aging per se. This finding refines previously reported observation of accelerated shrinkage in the HC of older adults affected by hypertension (Raz et al., 2005). The impacts of vascular factors on hippocampal subfield integrity have been largely overlooked in previous studies (except Wu et al., 2008) and should be examined more thoroughly as vascular health contributes substantially to brain aging. Unlike Mueller et al. (2008) and Mueller and Weiner (2009), but in agreement with Mueller et al. $(2007,2010)$, we found no significant age differences in the CA3-4\&DG volume. This discrepancy might stem from multiple factors, among them lack of carriers of a risky ApoEe4 allele, in whom the volume of that region may be particularly reduced (Mueller et al., 2008; Mueller and Weiner, 2009) and insufficient statistical power. In the absence of genetic data, we cannot ascertain that attribution.

The neurobiological meaning of the observed regional differences, as of in vivo MRI-based measures in general, is unclear. Only a handful of postmortem studies examined age differences in $\mathrm{HC}$ sub-regions and the findings are contradictory. In some samples, CA1 counts do not differ with age (West, 1993), whereas others reported strong age-related declines (Simic et al., 1997). Notably, neither study reported age differences in DG and CA3, in accord with our findings.

In examining the role of $\mathrm{HC}$ in binding stimulus elements into a unitary representation, rodent studies and neurocomputational models have stressed the role of the DG and its projections into the CA3 as neural substrates of pattern separation (O'Reilly and McClelland, 1994; Treves and Rolls, 1994). Pattern separation is believed to support the formation of discrete representations in memory by orthogonalizing cortical inputs as they enter the early 


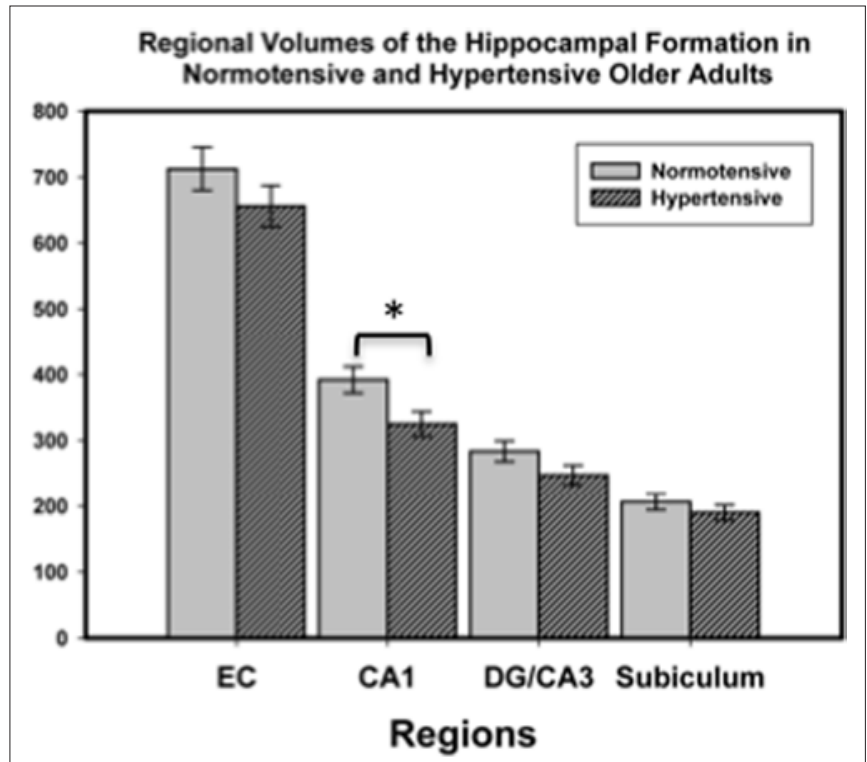

FIGURE 3 | Hippocampal subfield volumes in normotensive and hypertensive older adults. Group differences in volume were reliable only for subfield CA1-2. Volumes were adjusted for intracranial volume (ICV) as detailed in the text. The bars represent SE of the mean.

stages of the HC circuitry and the associated pathways. To encode and store overlapping inputs, the $\mathrm{HC}$ uses pattern separation by activating a sparse network of selective units, whereas to retrieve the information encoded in such sparse manner, it uses pattern completion (O'Reilly and Rudy, 2001). The fine balance between pattern separation and pattern completion is maintained by CA3 circuits of the HC (O'Reilly and McClelland, 1994) and allows formation of associations among encoded inputs and their retrieval as newly formed units, i.e., binding (Rolls, 1996). Recent fMRI findings support this view as they reveal activation consistent with a strong bias toward pattern separation in, and limited to, the CA3/ DG (Bakker et al., 2008).

It is plausible, therefore, that the observed link between CA3$4 \& \mathrm{DG}$ volume and increased FA rate reflects individual differences in maintaining pattern separation-pattern completion equilibrium. Rejection of familiar lures (a CA3/DG activator observed by Bakker et al., 2008) relies on adequate representation of differences between the stimuli. These conjectures are in accord with the model of Wilson et al. (2006), who suggested that, with aging, information processing in the DG and CA3 sub-regions is biased toward maintaining the representation of familiar contexts at the cost of weakened processing of new information. Pattern separation and completion may be especially challenging for the older brain characterized by a progressively reduced signal-to-noise ratio (Crossman and Szafran, 1956; Layton, 1975; Li et al., 2006). Indeed, older adults experience a specific difficulty in spatial pattern separation (Stark et al., 2010) and show increased interference among similar memory representations (Toner et al., 2009). Specificity of the CA3/DG involvement in memory is also supported by a recent report of a correlation between integrity of the PP, the major connection between EC and DG/CA3 and mem-
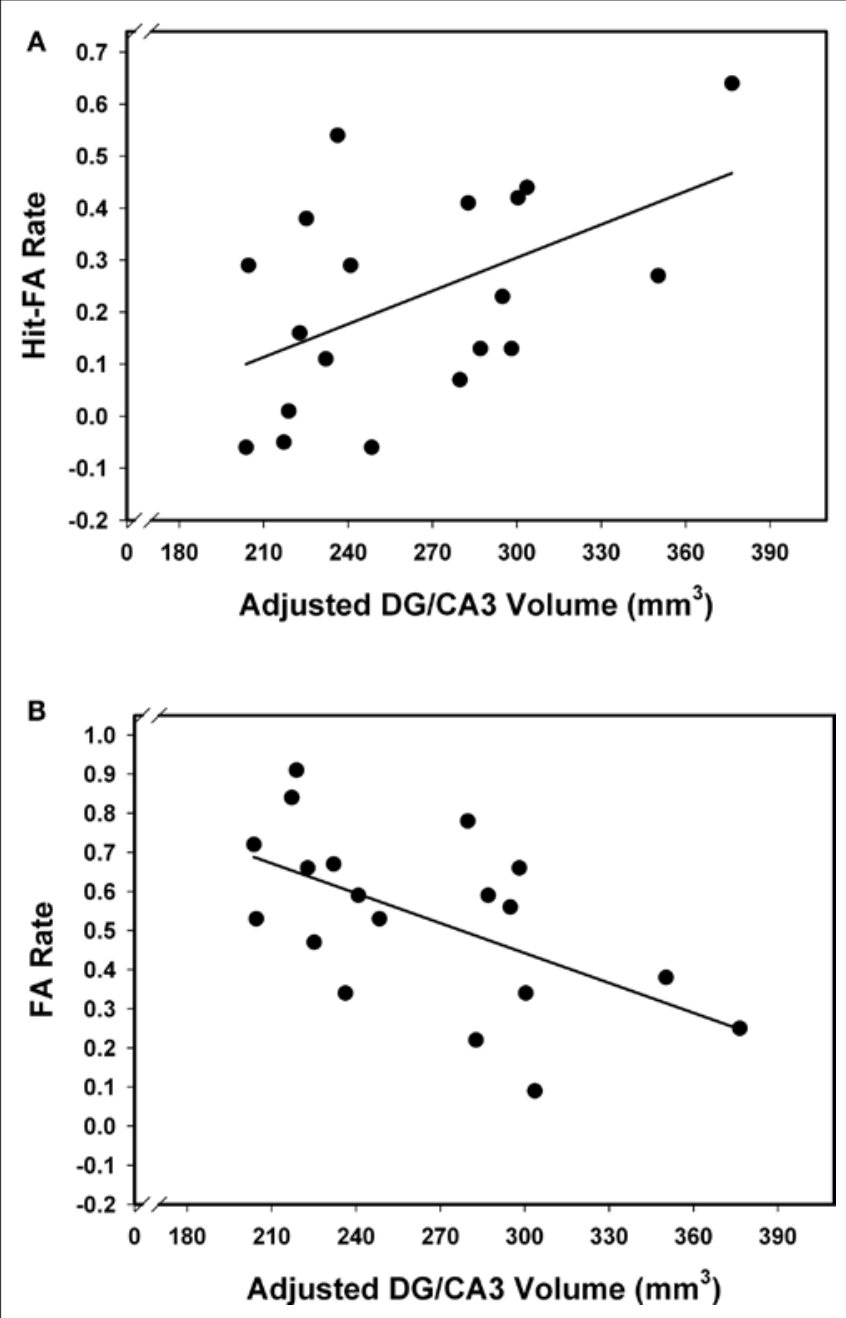

FIGURE 4 | Relation between memory performance and CA3-4\&DG volume. Larger CA3-4\&DG is associated with better memory performance (A), and in particular, lower FA rate (B).

ory performance (Yassa et al., 2010a). No such links were observed between PP and other cognitive measures or between memory and integrity of the alveus, a major pathway between EC and CA1.

It is intriguing that the only region associated with memory performance was the one that included the DG, the established site of adult neurogenesis (Altman and Das, 1965; Gould and Gross, 2002; Kempermann et al., 2004). Although there is no consensus on its function, adult neurogenesis has been proposed as a mechanism for preventing catastrophic interference while acquiring information in new environments (e.g., Wiskott et al., 2006), and adult-born neurons at different stages of maturity seem to enhance pattern separation differentially (Aimone et al., 2010). Catastrophic interference among newly acquired items may indeed be the ultimate expression of failed pattern separation. Specifically, individual differences in neurogenesis may contribute to individual differences in pattern separation, which in turn may influence the ability to reject rearranged pairs in the associative memory task. Studies with larger 
Table 1 | Correlations between $\mathrm{HC}$ subfield volumes, memory performance, and cognitive measures in older adults.

$\begin{aligned} & \text { Entorhinal } \\ & \text { cortex }\end{aligned}$
CA1-2

\begin{tabular}{|c|c|c|c|c|}
\hline \multicolumn{5}{|c|}{ MEMORY PERFORMANCE } \\
\hline Hit - FA & 0.17 & 0.34 & -0.30 & $0.50^{*}$ \\
\hline Hit rate & 0.09 & -0.12 & -0.33 & -0.18 \\
\hline FA rate & -0.11 & -0.39 & 0.10 & $-0.57^{*}$ \\
\hline \multicolumn{5}{|c|}{ COGNITIVE MEASURES } \\
\hline MMSE & -0.26 & $0.48^{*}$ & 0.24 & 0.32 \\
\hline Digit symbol & -0.12 & 0.31 & -0.30 & 0.31 \\
\hline
\end{tabular}

${ }^{*} p<0.05$.

and age-heterogeneous samples are needed to delineate the relation between associative memory and CA3-4\&DG. These studies should vary degree of redundancy among stimuli in the memory set to parametrically assess whether the ability to reject lures depends on the integrity of the CA3-4\&DG.

We also found that smaller CA1-2 volume was associated with reduced MMSE, a broad marker of cognitive status and a widely used screening instrument for dementia (Folstein et al., 1975), and the association was significant in spite of a narrow MMSE range. Our finding suggests that previously reported association between $\mathrm{HC}$ volume and MMSE in AD and mild cognitive impairment (e.g., Yavuz et al., 2007; Fjell et al., 2009), may stem from shrinkage of the CA1-2. It is unclear whether this finding reflects the admixture of undiagnosed dementia cases in our sample. In some (but not all) samples, CA1 appears as the focal point of neuronal loss in AD (West et al., 2004; but see Simic et al., 1997). Neuropathological studies found that neuronal loss, plaques, and tangles in the CA1 sub-regions (and subiculum) were associated with the extent of cognitive impairment in AD (Hyman et al., 1984). However, with an MMSE score at or above 26 , the likelihood ratio for dementia is very low (0.06-0.10; Siu, 1991), and it is unlikely that our finding reflects presence of preclinical AD. Moreover, using more stringent MMSE cut-offs did not alter the results.

Our findings suggest that age differences observed in this study and in the extant literature may be due to confounding age with agerelated vascular risk. As CA1 is particularly sensitive to hypoxia and ischemia (Zola-Morgan et al., 1986), history of vascular risk and vascular disease may be at least as strong a contributor to age differences as the insipient AD. It is also plausible that the admixture of hypertensive participants drove the association between CA1 volume and MMSE, but given the small number of hypertensive participants, a separate group analysis was not statistically feasible. Nonetheless, our findings underscore the necessity of considering vascular risk in studies of normal aging.

\section{REFERENCES}

Aimone, J. B., Deng, W., and Gage, F. H. (2010). Adult neurogenesis: integrating theories and separating functions. Trends Cogn. Sci. 14, 325-337.
Altman, J., and Das, G. D. (1965). Autoradiographic and histological evidence of postnatal hippocampal neurogenesis in rats. J. Comp. Neurol. $124,319-335$.

On a methodological note, our tracing method differed slightly from that of Mueller and colleagues. Insufficient anatomical boundary visualization compelled us to include the CA2 with the CA1 region. The difficulty in defining the CA2 region is reflected in the lower inter-rater reliabilities of its volume in Mueller et al. (2007). We also extended the tracing range of the ERC rostrally, which was made possible by the higher contrast-to-noise ratio of the PD-weighting and averaging across four acquisitions. As other researchers studying regional volumetry (Mueller et al., 2007, 2010; Yassa et al., 2010b) or functional activity (Bakker et al., 2008) of the HC, we had to combine DG and CA3 into one region of interest. The current state of technology does not yet allow clear demarcation of DG and CA3 borders. Notably, our measures were characterized by high inter-rater reliability as intra-class correlations employed here were computed under the assumption of random tracers. In the near future, highfield magnets are likely to enable clearer separation between DG and CA3 and increase the specificity in correlations between local HC structures and specific aspects of memory (Boretius et al., 2009).

The observed difference in delay between behavioral testing and scanning for $\mathrm{HC}$ volume measurements may have potentially affected the results. This is a limitation of this study. However, assuming that the individual rank order of memory scores and hippocampal volume does not change substantially across the scantest delay despite changes in magnitude, this limitation would not introduce a spurious relationship. To gauge the effect of variable delay on the findings we also tested for an effect of delay on the observed associations and found similar results. Nevertheless, our findings need to withstand scrutiny of future replications.

In sum, we report preliminary results supporting an association between increased likelihood of false recognition memory and reduced CA3/DG volume among older adults. We also observed an association between the volume of CA1 and a general cognitive index, thus suggesting a possibility of dissociation between functions of hippocampal regions. These findings are conceptually intriguing given the prominent role of DG in pattern separation and neurogenesis, and a possible role of CA1 in more general cognitive functions. Although the small sample size of this study may be of concern, the fact that the effects survived the bootstrapping tests argues for their robustness. Nonetheless, future studies with larger samples and memory data from younger individuals is necessary to determine if the observed associations are unique to old age or present throughout the lifespan.

\section{ACKNOWLEDGMENTS}

This work was conducted within the project "Cognitive and Neuronal Dynamics of Memory across the Lifespan (CONMEM)" at the Center for Lifespan Psychology, Max Planck Institute for Human Development, Berlin, Germany. This study was supported in part by the Max Planck Society, the German Research Foundation (DFG, HE 3347/3-1), the Alexander von Humboldt Foundation, and the National Institute of Health (R37 AG011230).

Apostolova,L.G., Mosconi, L., Thompson, P. M., Green, A. E., Hwang, K. S., Ramirez,A., Mistur, R., Tsui, W.H., and de Leon, M. J. (2010). Subregional hippocampal atrophy predicts Alzheimer's dementia in the cognitively normal. Neurobiol. Aging 31, 1077-1088.

Bakker, A., Kirwan, C. B., Miller, M., and Stark, C. E. (2008). Pattern separation in the human hippocampal 
CA3 and dentate gyrus. Science 319, 1640-1642.

Bartsch, T., Schonfeld, R., Muller, F. J., Alfke, K., Leplow, B.,Aldenhoff, J., Deuschl, G., and Koch, J.M. (2010). Focal lesions of human hippocampal CA1 neurons in transient global amnesia impair place memory. Science 328, 1412-1415.

Boretius, S., Kasper, L., Tammer, R., Michaelis, T., and Frahm, J. (2009). MRI of cellular layers in mouse brain in vivo. Neuroimage 47, 1252-1260.

Burggren, A. C., Zeineh, M. M., Ekstrom, A. D., Braskie, M. N., Thompson, P. M., Small, G. W., and Bookheimer, S.Y. (2008). Reduced cortical thickness in hippocampal subregions among cognitively normal apolipoprotein E e4 carriers. Neuroimage 41, 1177-1183.

Chételat, G., Fouquet, M., Kalpouzos, G., Denghien, I., De la Sayette, V., Viader, F., Mézenge, F., Landeau, B., Baron, J. C., Eustache, F., and Desgranges, B. (2008). Three-dimensional surface maping of hippocampal atrophc progression from $\mathrm{MCI}$ to $\mathrm{AD}$ and over normal aging as assessed using voxel-based morphometry. Neuropsychologia 46, 1721-1731.

Crossman, E. R. F. W., and Szafran, J. (1956). Changes with age in the speed of information-intake and discrimination. Experientia Suppl. 4, 128-135.

Duvernoy, H. M. (2005). The Human Hippocampus: Functional Anatomy, Vascularization and Serial Sections with MRI. Berlin: Springer.

Fjell, A. M., Amlien, I. K., Westlye, L. T., and Walhovd, K. B. (2009). Minimental state examination is sensitive to brain atrophy in Alzheimer's disease. Dement. Geriatr. Cogn. Disord. $28,252-258$.

Folstein, M. F., Folstein, S. E., and McHugh, P. R. (1975). Mini-mental state: a practical method for grading the cognitive state of patients for the clinician. J. Psychiatr. Res. 12, 189-198.

Franklin, S. S., Gustin, W. T., Wong, N. D., Larson, M. G., Weber, M.A., Kannel, W. B., and Levy, D. (1997). Hemodynamic patterns of age-related changes in blood pressure. The Framingham Heart Study. Circulation 96, 308-315.

Gould, E., and Gross, C. G. (2002). Neurogenesis in adult mammals: some progress and problems. J. Neurosci. 22, 619-623.

Hyman, B. T., Van Hoesen, G. W., Damasio, A. R., and Barnes, C. L. (1984). Alzheimer's disease: cellspecific pathology isolates the hippocampal formation. Science 225 , 1168-1170.

Jack, C. R. J., Petersen, R. C., Xu, Y., O’Brien, P. C., Smith, G. E., Ivnik, R. J., Boeve, B. F., Tangalos, E. G., and Kokmen, E. (2000). Rate of hippoc- ampal atrophy correlate with change in clinical status in aging and AD. Neurology 55, 484-489.

Jacoby, L. L., and Rhodes, M. G. (2006). False remembering in the aged. Curr. Dir. Psychol. Sci. 15, 49-53.

Kempermann, G., Jessberger, S., Steiner, B., and Kronenberg, G. (2004). Milestones of neuronal development in the adult hippocampus. Trends Neurosci. 27, 447-452.

Layton, B. (1975). Perceptual noise and aging. Psychol. Bull. 82, 875-883.

Levy, B. I., Schiffrin, E. L., Mourad, J. J., Agostini, D., Vicaut, E., Safar, M. E., and Struijker-Boudier, H. A. (2008). Impaired tissue perfusion: a pathology common to hypertension, obesity, and diabetes mellitus. Circulation 118 , 968-976.

Li, S.-C., Von Oertzen, T., and Lindenberger, U. (2006). A neurocomputational model of stochastic resonance and aging. Neurocomputing 69, 1553-1560.

Lister, J. P., and Barnes, C. A. (2009). Neurobiological changes in the hippocampus during normative aging. Arch. Neurol. 66, 829-833.

Mueller, S. G., Schuff, N., Raptentsetsang, S., Elman, J., and Weiner, M. W. (2008). Selective effect of Apo e4 on CA3 and dentatein normal aging and Alzheimer's disease using high resolution MRI at 4 T. Neuroimage 42, 42-48.

Mueller, S. G., Schuff, N., Yaffe, K., Madison, C., Miller, B., and Weiner, M. W. (2010). Hippocampal atrophy patterns in mild cognitive impairment and Alzheimer's disease. Hum. Brain Mapp. 31, 1339-1347.

Mueller, S. G., Stables, L., Du, A. T., Schuff, N., Truran, D., Cashdollar, N., and Weiner,M.W.(2007). Measurement of hippocampal subfields and age-related changes with high resolution MRI at 4 T. Neurobiol. Aging 28, 719-726.

Mueller, S. G., and Weiner, M. W. (2009). Selective effect of age, Apo e4, and Alzheimer's disease on hippocampal subfields. Hippocampus 19, 558-564.

Old, S. R., and Naveh-Benjamin, M. (2008). Differential effects of age on item and associative measures of memory: a meta-analysis. Psychol. Aging 23, 104-118.

O'Reilly, R. C., and McClelland, J. L. (1994). Hippocampal conjunctive encoding, storage, and recall: avoiding a trade-off. Hippocampus 4, 661-682.

O’Reilly, R. C., and Rudy, J. W. (2001). Conjunctive representations in learning and memory: principles of cortical and hippocampal function. Psychol. Rev. 108, 311-345.

Raz, N., Ghisletta, P., Rodrigue, K. M., Kennedy, K. M., and Lindenberger, U. (2010). Trajectories of brain aging in middle-aged and older adults: regional and individual differences. Neuroimage 51, 501-511.

Raz, N., Lindenberger, U., Rodrigue, K.M., Kennedy, K. M., Head, D., Williamson, A., Dahle, C., Gerstorf, D., and Acker, J.D. (2005). Regional brain changes in aging healthy adults: general trends, individual differences, and modifiers. Cereb. Cortex 15, 1676-1689.

Raz, N., and Rodrigue, K. M. (2006) Differential aging of the brain: patterns, cognitive correlates, modifiers. Neurosci. Biobehav. Rev. 30, 730-748.

Rolls, E. T. (1996). A theory of hippocampal function in memory. Hippocampus 6, 601-620.

Shing, Y. L., Werkle-Bergner, M., Li, S.-C., andLindenberger,U.(2008).Associative and strategic components of episodic memory: a lifespan dissociation. J. Exp. Psychol. Gen. 137, 495-513.

Shing, Y. L., Werkle-Bergner, M., Li, S.-C., and Lindenberger, U. (2009). Committing memory errors with high confidence: older adults do but children don't. Memory 17, 169-179.

Shrout,P.E., and Fleiss, J.L. (1979). Intraclass correlations: uses in assessing raters reliability. Psychol. Bull. 86, 420-428.

Simic, G., Kostovic, I., Winblad, B., and Bogdanovic, N. (1997). Volume and number of neurons of the human hippocampal formation in normal aging and Alzheimer's disease. J. Comp. Neurol. 379, 482-494.

Siu, A. L. (1991). Screening for dementia and investigating its causes. Ann. Intern. Med. 115, 122-132.

Small, S. A., Chawla, M. K., Buonocore, M., Rapp, P., and Barnes, C. A. (2004) Imaging correlates of brain function in monkeys and rats isolates a hippocampal subregion differentially vulnerable to aging. Proc. Natl. Acad. Sci. U.S.A. 101, 7181-7186.

Snodgrass, J. G., and Corwin, J. (1988). Pragmatics of measuring recognition memory: applications to dementia and amnesia. J. Exp. Psychol. Gen. 117 34-50.

Spencer, W. D., and Raz, N. (1995) Differential effects of aging on memory for content and context: a meta-analysis. Psychol. Aging 10 527-539.

Squire, L. R. (1987). Memory and Brain New York: Oxford University Press.

Stark, S. M., Yassa, M. A., and Stark, C. E. (2010). Individual differences in spatial pattern separation performance associated with healthy aging in humans. Learn. Mem. 17, 284-288.

Toner, C. K., Pirogovsky, E., Kirwan, C. B., and Gilbert, P. E. (2009). Visual object pattern separation deficits in nondemented older adults. Learn. Mem. 16, 338-342.
Treves, A., and Rolls, E. T. (1994). Computational analysis of the role of the hippocampus in memory. Hippocampus 4, 374-391.

Van Petten, C. (2004). Relationship between hippocampal volume and memory ability in healthy individuals across the lifespan: review and meta-analysis. Neuropsychologia 42, 1394-1413.

Verhaeghen, P., Marcoen, A., and Goosens, L. (1993). Facts and fiction about memory aging: a quantitative integration of research findings. J. Gerontol. Psychol. Sci. 48, 157-171.

Wechsler, D. (1955). Wechsler Adult Intelligence Scale Manual. New York: Psychological Corporation.

West, M. J. (1993). Regionally specific loss of neurons in the aging human hippocampus. Neurobiol. Aging 14, 287-293.

West, M. J., Kawas, C. H., Stewart, W. F., Rudow, G. L., and Troncoso, J. C. (2004). Hippocampal neurons in preclinical Alzheimer's disease. Neurobiol. Aging 25, 1205-1212.

Wilson, I. A., Gallagher, M., Eichenbaum, H., and Tanila, H. (2006). Neurocognitive aging: prior memories hinder new hippocampal encoding. Trends Neurosci. 29, 662-670.

Wiskott,L., Rasch,M.J., and Kempermann, G. (2006). A functional hypothesis for adult hippocampal neurogenesis: avoidance of catastrophic interference in the dentate gyrus. Hippocampus 16, 329-343.

Wu, W., Brickman, A. M., Luchsinger, J., Ferrazzano, P., Pichiule, P., Yoshita, M., Brown, T., DeCarli, C., Barnes, C. A., Mayeux, R., Vannucci, S. J., and Small, S. A. (2008). The brain in the age of old: the hippocampal formation is targeted differentially by diseases of late life. Ann. Neurol. 64, 698-706.

Yassa, M.A., Muftuler, L. T., and Stark, C.E. (2010a). Ultrahigh-resolution microstructural diffusion tensor imaging reveals perforant path degradation in aged humans in vivo. Proc. Natl. Acad. Sci. U.S.A. 107, 12687-12691.

Yassa, M.A., Stark, S. M., Bakker,A.,Albert, M. S., Gallagher, M., and Stark, C. E. L. (2010b). High-resolution structural and functional MRI of hippocampal $\mathrm{CA} 3$ and dentate gyrus in patients with amnestic mild cognitive impairment. Neuroimage 51, 1242-1252.

Yavuz, B. B., Ariogul, S., Cankurtaran, M., Oguz, K. K., Halil, M., Dagli, N., and Cankurtaran, E. S. (2007). Hippocampal atrophy correlates with the severity of cognitive decline. Int. Psychogeriatr. 19, 767-777.

Zola-Morgan, S., Squire, L. R., and Amaral, D. G. (1986). Human amnesia and the medial temporal region: endur- 
ing memory impairment following a bilateral lesion limited to field CA1 of the hippocampus. J. Neurosci. 6, 2950-2967.

Conflict of Interest Statement: The authors declare that the research was conducted in the absence of any commercial or financial relationships that could be construed as a potential conflict of interest.

Received: 13 December 2010; accepted: 14 January 2011; published online: 04 February 2011.

Citation: Shing YL, Rodrigue KM, Kennedy KM, Fandakova Y, Bodammer
$N$, Werkle-Bergner $M$, Lindenberger $U$ and Raz N (2011) Hippocampal subfield volumes: age, vascular risk, and correlation with associative memory. Front. Ag. Neurosci. 3:2. doi: 10.3389/ fnagi.2011.00002

Copyright (c) 2011 Shing, Rodrigue, Kennedy, Fandakova, Bodammer,
Werkle-Bergner, Lindenberger and Raz. This is an open-access article subject to an exclusive license agreement between the authors and Frontiers Media SA, which permits unrestricted use, distribution, and reproduction in any medium, provided the original authors and source are credited. 